\title{
Moving Least Squares Multiresolution Surface Approximation
}

\author{
Boris Mederos Luiz Velho Luiz Henrique de Figueiredo \\ IMPA-Instituto de Matemática Pura e Aplicada \\ Estrada Dona Castorina 110, 22461-320 Rio de Janeiro, RJ, Brazil \\ boris@impa.br lvelho@impa.br lhf@impa.br
}

\begin{abstract}
We describe a new method for surface reconstruction based on unorganized point clouds without normals. We also present a new algorithm for refining the initial triangulation. The output of the method is a refined triangular mesh with points on the moving least squares surface of the original point cloud.
\end{abstract}

Keywords: multi-resolution; surface reconstruction; $k$-nearest neighbors; moving least squares.

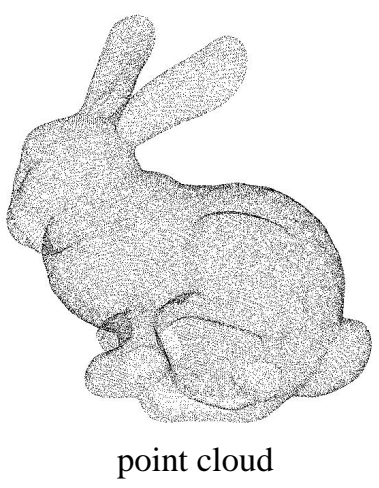

\section{Introduction}

We consider the problem of surface reconstruction and refinement from scattered data points without normals. Several algorithms are known for this important problem [4-13], including a number of recent algorithms with theoretical guarantees [4-7]. Those algorithms use a 3D Delaunay triangulation of the original point cloud to compute a triangular surface mesh. Computing the Delaunay triangulation can be slow and susceptible to numerical errors.

Gopi, Krishnan, and Silva [12] proposed an algorithm based on Differential Geometry that projects the neighborhood of each sample point on a tangent plane, computes the 2D Delaunay triangulation of this projected neighborhood, and lifts it to $3 \mathrm{D}$.

Hoppe et al. [10] estimate a tangent plane at each sample point using its $k$-nearest neighbors and use the distance to the plane of the nearest sample as an estimative of the signed distance function to the surface. The zero set of this distance function is extracted using the marching cubes algorithm.

Other algorithms $[8,9,13]$ use a greedy approach. The ball pivoting algorithm [9] rolls a ball over the sample points; it requires the normal to the surface at each sample point, but it can create artifacts. Boissonnat [8] starts

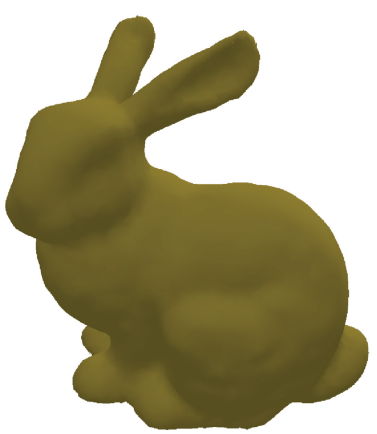

triangulation

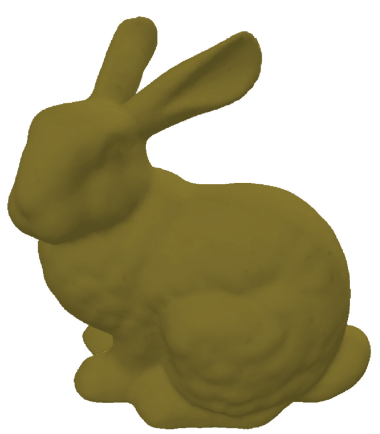

refinement by finding an initial edge $p q$ in the triangulated reconstruction; he then computes a tangent plane around the edge, projects the $k$-nearest neighbors of both vertices onto this plane, and determines the point $r$ that maximizes the angle $\angle \bar{p} \bar{r} \bar{q}$ (where the bar represents projected points on the tangent plane). This point $r$ determines a surface triangle prq. The process is repeated for each border edge, resulting in a triangulated surface. Boyer [13] proposes an incremental algorithm over the 3D Delaunay triangulation of the samples, and relies on regular interpolants.

Our algorithm uses a different approach: it computes a set of representative points near of the original point cloud and triangulates these representative points using a new incremental algorithm based on $k$-nearest neighbors. This is somewhat similar to what is done in other greedy algorithms [8, 9, 13]: For each border edge, the algorithm uses angle criteria to select a point to make a triangle with the edge. The initial triangulation is refined using a new method based on moving least squares operators [1-3]. Our algorithm does not need Delaunay triangulations and it can handle surfaces with borders.

Section 2 describes in detail the four steps of our method: clustering, reduction, triangulation, and refinement. These steps are illustrated above. Section 3 discusses several examples of the method in action. 

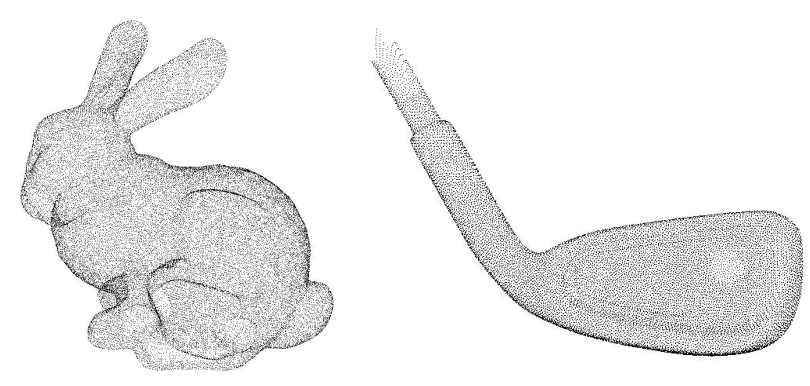

Figure 1: Point clouds

\section{Our Method}

Starting from a point cloud $\mathcal{Q} \subseteq \mathbf{R}^{3}$ that samples an unknown $C^{1}$ surface $\mathcal{S}$, our method produces a mesh with points near the moving least squares surface of $\mathcal{Q}$ [1-3]. The method has four steps:

1. Clustering: Split the point cloud into a set of clusters.

2. Reduction: Compute a representative point of each cluster on the moving least squares surface of its $k$-nearest neighbors.

3. Triangulation: Build a triangulated surface over the set of representative points.

4. Refinement: Refine the initial mesh into a finer mesh that is adapted to the geometry of the unknown surface $\mathcal{S}$.

We shall now describe each of these steps in detail.

\subsection{Clustering}

The goal of this first step is to partition the original set of points $\mathcal{Q}$ into a finite set of clusters, such that the curvature of the original surface $\mathcal{S}$ varies a little within each cluster. Since $\mathcal{S}$ is not known, its curvature must be estimated from the sample points $\mathcal{Q}$.

We use a hierarchical clustering method based on a BSP tree [3]. Each node in this tree contains a subset $\mathcal{P}=\left\{p_{1}, \ldots, p_{n}\right\}$ of the original point cloud $\mathcal{Q}$. We use the covariance matrix $C$ of $\mathcal{P}$ to decide whether or not to subdivide the node:

$$
C=\left[\begin{array}{c}
p_{1}-\bar{p} \\
p_{2}-\bar{p} \\
\vdots \\
p_{n}-\bar{p}
\end{array}\right]^{T}\left[\begin{array}{c}
p_{1}-\bar{p} \\
p_{2}-\bar{p} \\
\vdots \\
p_{n}-\bar{p}
\end{array}\right], \quad \bar{p}=\frac{1}{n} \sum_{i=1}^{n} p_{i} .
$$

Since $C$ is a symmetric positive semi-definite $3 \times 3$ matrix, its three eigenvalues are real and we can order them: $\lambda_{1} \leq \lambda_{2} \leq \lambda_{3}$. These eigenvalues measure the variation of the points in $\mathcal{P}$ along the directions of their corresponding eigenvectors $v_{1}, v_{2}, v_{3}$.
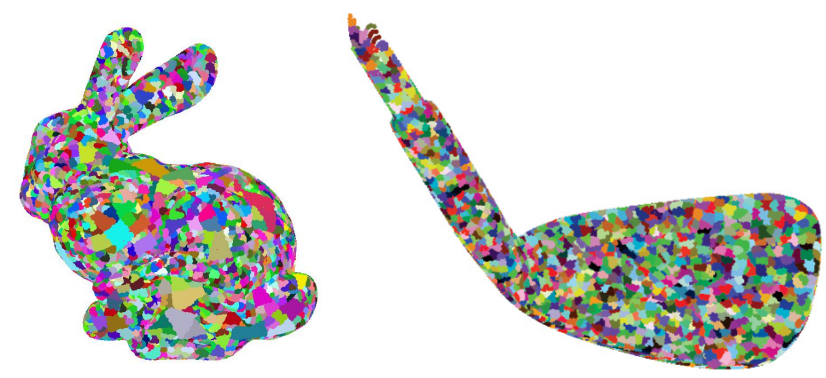

Figure 2: Clusters

The eigenvectors $v_{2}$ and $v_{3}$ define the directions of highest variation and define a regression plane $\Pi$ for $\mathcal{P}$. The eigenvector $v_{1}$ is normal to $\Pi$ and its eigenvalue $\lambda_{1}$ measures the variation of the points in $\mathcal{P}$ with respect to the plane $\Pi$. So, small values of $\lambda_{1}$ mean that all points in $\mathcal{P}$ are approximately on $\Pi$. Hence, the ratio

$$
\sigma=\frac{\lambda_{1}}{\lambda_{1}+\lambda_{2}+\lambda_{3}}
$$

is a good measure of the flatness of the point set $\mathcal{P}$ and can be used as an estimate for the curvature of $\mathcal{S}$ around $\mathcal{P}$.

We use this flatness measure to define subdivision criteria for the BSP tree. A node is divided in two when both conditions below are satisfied:

1. The ratio $\sigma$ is larger than a user-defined tolerance $\sigma_{\max }$;

2. The number $n$ of points in $\mathcal{P}$ is larger than a userdefined value $n_{\text {min }}$.

The nodes are divided by classifying the points of $\mathcal{P}$ with respect to the regression plane $\Pi$. The points of $\mathcal{P}$ that are in the same side of $\Pi$ will form a new node. The leaves of the BSP tree will be the clusters. Figure 2 shows the clusters computed for the point clouds shown in Figure 1.

\subsection{Reduction}

We find a representative point for each cluster using a new method based on the moving least squares theory [1-3]. The moving least squares surface of a set of points $R$ near the surface $\mathcal{S}$ is the set of fixed points of a projection operator $\Psi(R, \cdot)$ defined in [1-3]:

$$
\operatorname{MLS}(R)=\left\{y \in \mathbf{R}^{3}: \Psi(R, y)=y\right\} .
$$

Given a point point $r \in \mathbf{R}^{3}$, we approximate $\Psi(\mathcal{Q}, r)$ by $\Psi(K, r)$, where $K$ is the set of $k$-nearest neighbors of $r$ in $\mathcal{Q}$ (see [3] for details).

To determine a representative point for each cluster, we start with the centroid $c$ of the set of points in the cluster. This point $c$ is not necessarily near $\mathcal{S}$ and so we move 

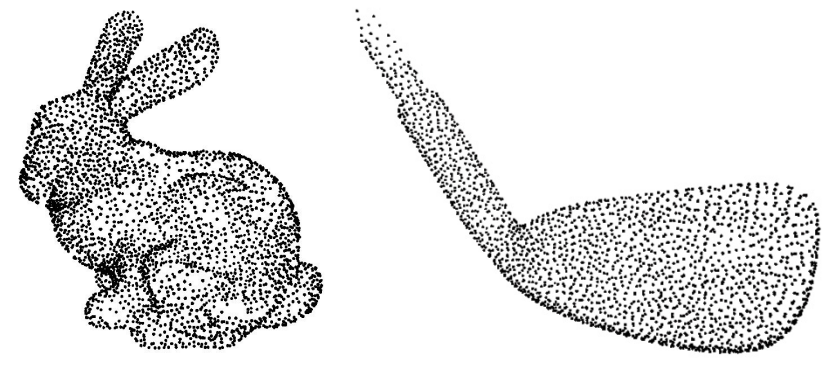

Figure 3: Representatives

it toward $\mathcal{S}$ and obtain the point $r=c+t_{\min } n_{c}$, where $n_{c}$ and $t_{\min }$ are explained below. The point $r$ is then projected onto the moving least squares surface $\operatorname{MLS}(\mathcal{Q})$ of the initial point cloud and we obtain the point $r_{p r o j}=\Psi(Q, r)$, which is selected to represent the cluster. Figure 3 shows the representatives for the clusters shown in Figure 2.

The vector $n_{c}$ used to define $r$ is the eigenvector associated to the smallest eigenvalue of a weighted covariance matrix $M$ over the set $K$ of $k$-nearest neighbors of $c$ in $\mathcal{Q}$. More precisely, $M$ is the $3 \times 3$ matrix whose entries are

$$
m_{i j}=\sum_{p \in K}\left(p_{i}-c_{i}\right)\left(p_{j}-c_{j}\right) \exp \left(\frac{-\|p-c\|^{2}}{h^{2}}\right),
$$

where $p=\left(p_{1}, p_{2}, p_{3}\right), c=\left(c_{1}, c_{2}, c_{3}\right)$, and $h$ is a parameter reflecting the spacing between neighboring points in $\mathcal{Q}$ (see $[1,2]$ for details). We use $h$ equal to three times the distance of $c$ to its near neighbor in $\mathcal{Q}$.

Using the direction $n_{c}$ as above, we compute

$$
t_{\text {min }}=\arg \min _{t} \sum_{p \in K}\left\|p-c-t n_{c}\right\|^{2} .
$$

We interpret this as finding the point $r=c+t_{\min } n_{c}$ on the line through $c$ in the direction of $n_{c}$ that is closest to the original surface.

\subsection{Triangulation}

The next step is to build a triangulated surface over the set of representative points. We do this using an incremental algorithm that introduces new features in the basic framework of previous incremental algorithms $[8,9,13]$.

The main idea behind the algorithm is to determine incrementally the restricted Delaunay triangles, because these triangles form a piecewise linear manifold homeomorphic to the original surface [4].

The algorithm computes a sequence of triangulated surfaces with border. At each step, it chooses a border edge, finds a new triangle associated to this edge, and updates the current surface. The algorithm maintains a half-edge data structure $H$ that represents the current surface and a list $L$ of half-edges that represents the current boundary. Here is a summary of the main steps:

Build a 3d-tree on the set of representatives.

Get an initial triangle and initialize $H$ and $L$ with it.

repeat

Remove a half edge $u v$ from $L$.

$u s v \leftarrow$ the triangle adjacent to $u v$ in $H$.

$P \leftarrow$ set of $k$-nearest neighbors of $u$.

$q \leftarrow$ GET_POINT( $u s v, u, P)$.

Insert $u q v$ into $H$ and its border edges into $L$.

until $L$ is empty.

The initial surface contains a single triangle. This triangle is inserted into $H$ and all its edges are inserted into $L$. To find the initial triangle, we select a point $p \in \mathcal{Q}$ and determine its nearest neighbor $q \in \mathcal{Q}$. These two points define the first border edge: $p q$. Among the $k$-nearest neighbors of $p$, we determine the point $r$ that maximizes the angle $\angle p r q$. The initial triangle $p r q$ is in the Delaunay triangulation of the $k$-nearest neighbors of $p$ (see [14], Lemma 3).

Once the initial surface has been found, we continue by removing edges from $L$ until it is empty. For each edge $u v$, we select a point $q$ among the $k$-nearest neighbors of $u$ to create a new triangle $u q v$, which is added to $H$ and $L$. (We use a $3 \mathrm{~d}$-tree built at the start of the algorithm to identify $k$-nearest neighbors.)

The number of edges of $u q v$ inserted in $L$ varies: it is zero when the triangle $u q v$ fills a hole in $H$; it is one when $u q$ or $v q$ are consecutive edges of $u v$ in the border of the current surface; otherwise, it is two. When $q$ is already in $H$, we have to join two connected components of the border or to split one component in two new connected components. We determine the point $q$ using the following algorithm:

\section{GET_POINT ( $u s v, u, P)$ :}

$d_{u} \leftarrow$ distance from $u$ to its nearest neighbor.

$d_{v} \leftarrow$ distance from $v$ to its nearest neighbor.

$d_{\min } \leftarrow \min \left\{d_{u}, d_{v}\right\}$.

repeat

$C_{\theta} \leftarrow$ set of points $t$ such that:

(i) the dihedral angle between the triangles usv and $u t v$ is in $[\pi-\theta, \pi+\theta]$;

(ii) Triangle $u t v$ does not violate the topology of $H$;

(iii) $\frac{\max \{d(u, t), d(v, t)\}}{d_{\min }}<\varepsilon_{1}$.

if $C_{\theta} \neq \emptyset$ then

Determine the set $C_{\theta}^{\prime}$ of points $q \in C_{\theta}$ with maximum angle $\angle u q v$.

$$
\text { else }
$$$$
\theta \leftarrow \theta+\varepsilon_{2} .
$$$$
\text { until } C_{\theta}^{\prime} \neq \emptyset \text { or } \theta>\frac{\pi}{2}+\varepsilon_{2}
$$

Here, $\theta, \varepsilon_{1}$, and $\varepsilon_{2}$ are user-defined tolerances. 
Condition (i) in this algorithm is based on the theorem below, which we prove in [14]:

Theorem 1 Let $F$ be a $\beta, r$-sampling of a surface $\mathcal{S}$ with $r<1 / 4$. Then the angle between two adjacent restricted Delaunay triangles sharing an edge is at least $\pi-2\left(\frac{2 r}{1-4 r}+\right.$ $\left.\arcsin \left(\frac{\sqrt{3} r}{1-r}\right)\right)$.

This theorem show that the dihedral angle between two adjacent restricted Delaunay triangles converges to $\pi$ as the sampling density increases (that is, as $r \rightarrow 0$ ). We start with an initial region $[\pi-\theta, \pi+\theta]$ with small $\theta$ to determine the set of point $C_{\theta}$ in this region that satisfy criteria (ii) and (iii). If $C_{\theta}$ is not empty, we find the point $q \in C_{\theta}$ with maximum angle $\angle u q v$; otherwise, we increase $\theta$ by $\varepsilon_{2}$ and repeat the process until we find a point $q$ or $\theta$ gets too large.

Condition (ii) is used to eliminate the points $t$ that are interior to $H$ and the points on the boundary of $H$ that would violate the topology if they were selected. When the point $t$ is on the boundary of $H$, we first fit a plane $\Pi$ to the set of points adjacent to $t$ in $H$ (the star of $t$ ) and project this start onto $\Pi$, resulting in a set of $2 \mathrm{~d}$ triangles $T$. If these triangles do not form a triangulation, that is, is two edges intersect, then we have a topology violation.

Condition (iii) is used to determine border edges, and is based on the following theorem, which we prove in [14]:

Theorem 2 Let $F$ be a $\beta, r$-sampling of a surface $\mathcal{S}$ with $r<1 / 3$. Let $T_{1}=u v t$ and $T_{2}=u v q$ be restricted Delaunay triangles sharing the edge uv. Then, the length of the longest edge of $T_{2}$ is at most $\frac{2 \beta}{1-3 r} d$, where $d=$ $\min \left\{d_{u}, d_{v}\right\}$ and $d_{u}$ (respectively, $d_{v}$ ) is the distance of $u$ (respectively, $v$ ) to its nearest neighbor.

This theorem shows that there is little variation between the length of the edges of two triangles adjacent to a interior edge. We observed that the length of edges of the adjacent triangles to a boundary edge are very different.

In practice, we have no way to estimate $\beta$ and $r$, and so we use a predefined value $\varepsilon_{1}$ as the constant $\frac{2 \beta}{1-3 r}$ and a point $t$ is eliminated if $\frac{\max \{d(u, t), d(v, t)\}}{d_{\min }}>\varepsilon_{1}$. If $C_{\theta}$ is empty, the edge $u v$ is a candidate border edge. If $C_{\theta}$ is empty for all $\theta$ tested, $u v$ is a border edge.

If $C_{\theta}$ is not empty, we compute the subset $C_{\theta}^{\prime}$ of points $q$ with maximum angle $\angle u q v$. We justify this criterion as follows: In $\mathbf{R}^{2}$, given a Delaunay triangle $u v s$, its adjacent Delaunay triangle $u v q$ is the one with maximum angle $\angle u q v$. Although this is a criterion for $\mathbf{R}^{2}$, we use it for surfaces because the surface normal varies little in the surface neighborhood of a point $u$ containing the vertex of the restricted Delaunay triangulation (see [4-6]); in other words, we can consider this neighborhood as flat.
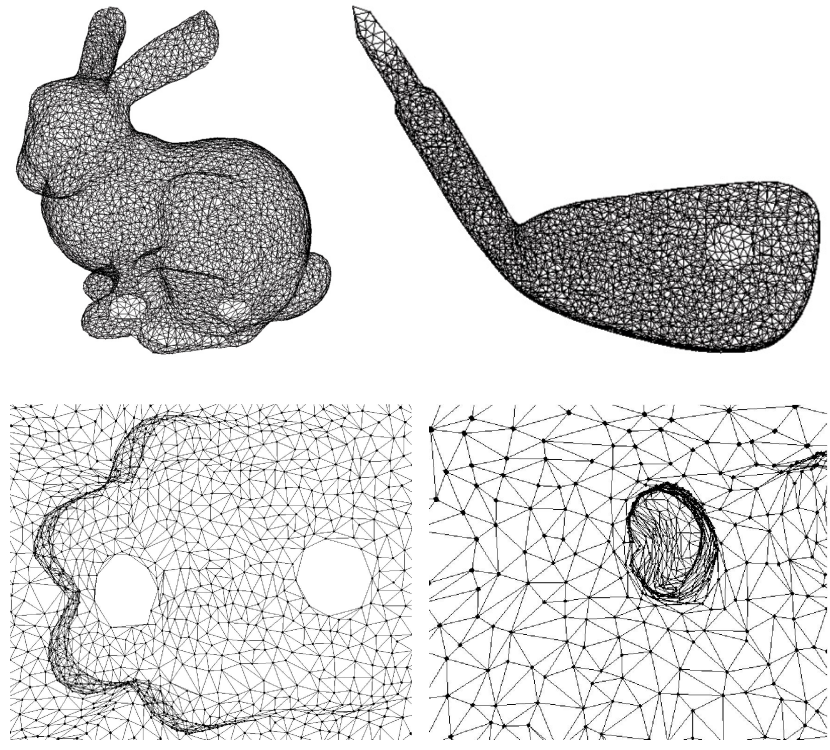

Figure 4: Triangulations

Figure 4 shows the triangulated surfaces over the set of representatives shown in Figure 3.

\subsection{Refinement}

The goal of this step is to refine the initial coarse triangulation into a finer triangulation adapted to the geometry of the unknown surface $\mathcal{S}$ from which the point cloud $\mathcal{Q}$ was sampled.

We propose the following method to refine an edge $u v$ : We compute the middle point of the edge. If this point is too far from the original point cloud $\mathcal{Q}$, it is projected onto the moving least squares surface and a new edge is added to each adjacent triangle of the edge $u v$, dividing each triangle into two new triangles. We repeat this process for each edge in the original triangulation. The details are discussed below.

More precisely, for each edge $u v$ we compute its midpoint $m=\frac{u+v}{2}$ and its normal

$$
n_{m}=\frac{\frac{n_{u}+n_{v}}{2}}{\left\|\frac{n_{u}+n_{v}}{2}\right\|}
$$

where $n_{u}$ and $n_{v}$ are the normals of $u$ and $v$ respectively, computed using the local triangulation (star) of each vertex.

Using the set $K$ of $k$-nearest neighbors of $m$ in the original points cloud, we minimize the following functional with respect to $t$ :

$\alpha=\min _{t} \sum_{p \in K}\left\|p-m-t \cdot n_{m}\right\|^{2}=-\frac{1}{k} \sum_{p \in K}(p-m) \cdot n_{m}$ 

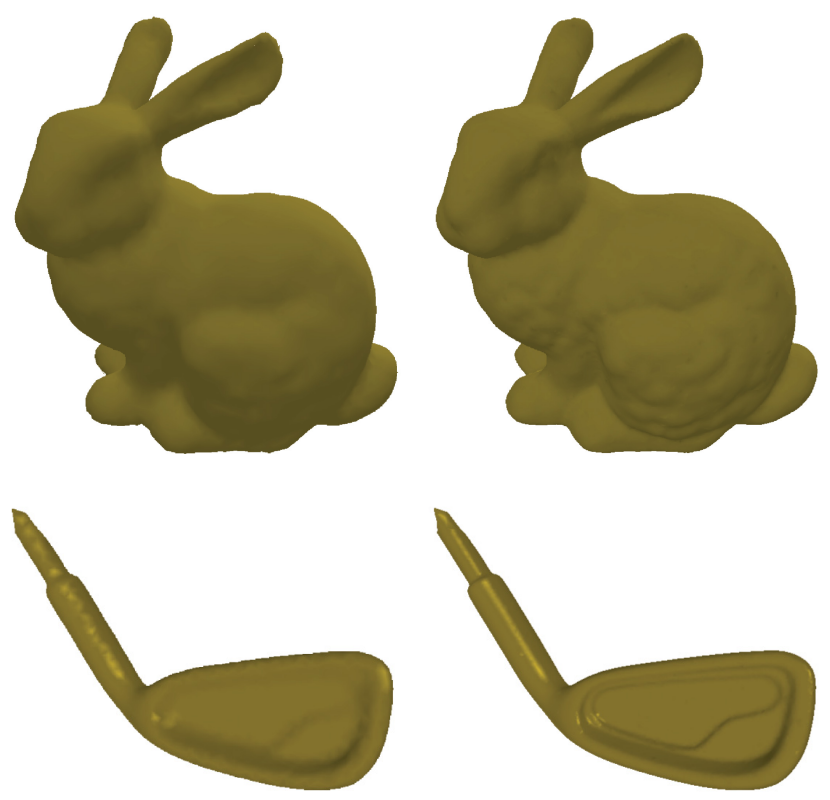

Figure 5: Refinement

As in the reduction step, this is interpreted as finding the point on the line through $m$ in the direction of $n_{m}$ that is closest to the original surface $\mathcal{S}$.

We use $\alpha$ as a measure of the need of refinement. If $\alpha$ is smaller than some predefined value $\varepsilon$, we do not refine the edge; otherwise, we project the point $m+\alpha \cdot n_{m}$ onto the moving least squares surface, producing the new point $\bar{m}=\Psi\left(\mathcal{Q}, m+\alpha \cdot n_{m}\right)$ and replace the triangles auv and $b u v$ adjacent to $u v$ with the new triangles $u \bar{m} a, v \bar{m} a$ and $u \bar{m} b, v \bar{m} b$, respectively. If the ratio between the length of the minimum edge and the maximum edge of the triangles $a u v$ or buv is too small, we do not divide the triangles.

This procedure is applied to all the old edges while the scalar $t$ is larger than $\varepsilon$. In the resulting mesh, we flip the diagonals of each quadrilateral formed by the pairs of adjacent triangles if the length of one diagonal (the common edge) is larger than the length of the other diagonal (the segment joining the opposite vertex).

The final result is a triangulated surface whose vertices are on the moving least squares approximation surface of the original points cloud. Figure 5 shows two refinement of the initial triangulations shown in Figure 4.

\section{Results}

Our algorithm has been implemented using CGAL [15] for computing the half edge data structure and the operations in the refinement. The algorithm has been tested on several data sets available on the internet $[16,17]$ using two refinement steps. The results are shown in Figures 6-9 (and

\begin{tabular}{|c|c|c|c|c|}
\hline Model & Clustering & Triangulation & Ref. 1 & Ref. 2 \\
\hline Bunny & $3.68 \mathrm{~s}$ & $22.48 \mathrm{~s}$ & $2.33 \mathrm{~s}$ & $7.9 \mathrm{~s}$ \\
$35933 \mathrm{p}$ & $4457 \mathrm{p}$ & $8895 \mathrm{t}$ & $31623 \mathrm{t}$ & $107363 \mathrm{t}$ \\
\hline Club & $1.36 \mathrm{~s}$ & $5.3 \mathrm{~s}$ & $1.11 \mathrm{~s}$ & $3.86 \mathrm{~s}$ \\
$16864 \mathrm{p}$ & $2103 \mathrm{p}$ & $4121 \mathrm{t}$ & $15037 \mathrm{t}$ & $51775 \mathrm{t}$ \\
\hline Cactus & $0.21 \mathrm{~s}$ & $1.58 \mathrm{~s}$ & $0.2 \mathrm{~s}$ & $0.68 \mathrm{~s}$ \\
$3337 \mathrm{p}$ & $404 \mathrm{p}$ & $782 \mathrm{t}$ & $2776 \mathrm{t}$ & $9614 \mathrm{t}$ \\
\hline Cat & $0.77 \mathrm{~s}$ & $6.02 \mathrm{~s}$ & $0.61 \mathrm{~s}$ & $2.06 \mathrm{~s}$ \\
$10000 \mathrm{p}$ & $1244 \mathrm{p}$ & $2453 \mathrm{t}$ & $29257 \mathrm{t}$ & $8535 \mathrm{t}$ \\
\hline Dragon & $62.43 \mathrm{~s}$ & $297.8 \mathrm{~s}$ & $27.22 \mathrm{~s}$ & $58.98 \mathrm{~s}$ \\
$437645 \mathrm{p}$ & $55694 \mathrm{p}$ & $109889 \mathrm{t}$ & $361455 \mathrm{t}$ & $810367 \mathrm{t}$ \\
\hline Horse & $4.67 \mathrm{~s}$ & $30.83 \mathrm{~s}$ & $3.17 \mathrm{~s}$ & $10.76 \mathrm{~s}$ \\
$48482 \mathrm{p}$ & $6164 \mathrm{p}$ & $12046 \mathrm{t}$ & $42860 \mathrm{t}$ & $144828 \mathrm{t}$ \\
\hline
\end{tabular}

Table 1: Results on a $1.8 \mathrm{Ghz}$ Pentium 4 with $512 \mathrm{Mb}$ of RAM running Linux ( $\mathrm{s}=$ seconds, $\mathrm{p}=$ points, $\mathrm{t}=$ triangles $)$

also in the previous figures). Table 1 shows the times (in seconds) taken in each step of the algorithm and the sizes (points and triangles) of the result models.

In the clustering step, the typical values for the userdefined parameters were $\sigma_{\max }=0.005$ and $n_{\min }=32$. In the triangulation algorithm, we obtain very good results using $\varepsilon_{1}=2.8$ for identifying boundary edges, $\theta=60^{\circ}$ for the initial angle, and $\varepsilon_{2}=15^{\circ}$ for the angle step. We also observed that the ratio $\frac{\max \{d(u, t), d(v, t)\}}{d_{\min }}$ is frequently larger than 3 in the border of the surface.

The pictures in this paper are available in full size and color at http: //www. impa.br/ boris/sib2003.html.

\section{Conclusion}

We presented a new algorithm for reconstruction and refinement of surface, giving as output a refined triangular mesh with points in the moving least squares surface of the original points cloud.

The new method proposed for computing representatives points produces a simplified sample on the moving least squares surface. The new triangulation algorithm does not need to compute 3D Delaunay triangulations, which in the worst case requires quadratic time and is prone to numerical errors. The new refinement method is fast and gives a fine triangular mesh adapted to the geometry of the unknown surface.

Acknowledgments. The authors are partially supported by $\mathrm{CNPq}$ research grants. The authors are members of Visgraf, the computer graphics laboratory at IMPA, which is sponsored by CNPq, FAPERJ, FINEP, and IBM Brasil. This work is part of Boris Mederos's doctoral thesis at IMPA.

\section{References}

[1] M. Alexa, J. Behr, D. Cohen-Or, S. Fleishman, D. Levin, C. Silva. Point set surfaces. IEEE Visualization 2001, pp. 21-28. 
[2] M. Alexa, J. Behr, D. Cohen-Or, S. Fleishman, D. Levin, C. Silva. Computing and rendering point set surfaces. IEEE Transactions on Visualization and Computer Graphics 9(1):3-15, 2003.

[3] M. Pauly, M. Gross, L. Kobbelt. Efficient simplification of point-sampled surfaces. IEEE Visualization 2002, pp. 163170.

[4] N. Amenta, M. Bern, M. Kanvisellis. A new Voronoi based surface algorithm. SIGGRAPH'98, pp. 415-421.

[5] N. Amenta, S. Choi. One-pass Delaunay filtering for homeomorphic 3D surface reconstruction. Technical Report TR9908, University of Texas at Austin, 1999.

[6] N. Amenta, S. Choi, R. Kolluri. The power crust. 6th ACM Symposium on Solid Modeling, pp. 249-260, 2001.

[7] J. D. Boissonnat, F. Cazals. Smooth surface reconstruction via natural neighbor interpolation of distance function. Computational Geometry 22(1-3):185-203, 2002.

[8] J. D. Boissonnat. Geometric structures for three-dimensional shape reconstruction. ACM Transactions on Graphics 3(4):266-289, 1984.

[9] F. Bernardini, J. Mittelman, H. Rushmeier, C. Silva, G. Taubin. The ball-pivoting algorithm for surface reconstruction. IEEE Transactions on Visualization and Computer Graphics 5(4):349-359, 1999.

[10] H. Hoppe, T. DeRose, T. Duchamp, J. McDonald, W. Stuetzle. Surface reconstruction from unorganized points. SIGGRAPH'92. pp. 71-78.

[11] D. Freedman. Efficient simplicial reconstruction of manifolds from their samples. IEEE Transaction on Pattern Analysis and Machine Intelligence 24(10):1349-1357, 2002.

[12] M. Gopi, S. Krishnan, C. Silva. Surface reconstruction based on lower dimensional localized Delaunay triangulation. Computer Graphics Forum 19(3):C467-C478, 2000.

[13] E. Boyer, S. Petitjean. Curve and surface reconstruction from regular and non regular point sets. Computational Geometry 19(2-3):101-126, 2001.

[14] B. Mederos, L. Velho, L. H. de Figueiredo. A Simple Algorithm for Multiresolution Surface Reconstruction. Technical Report TR03-03, Instituto de Matemática Pura e Aplicada, 2003. http://www.visgraf.impa.br/ RefBib/Data/PS_PDF/tr0303/

[15] CGAL: Computational Geometry Algorithms Library. www.cgal.org

[16] Large Geometric Models Archive at Georgia Tech. www.cc.gatech.edu/projects/large_models/

[17] Hugues Hoppe's home page. www.research.microsoft.com/ ${ }^{\text {hoppe/ }}$
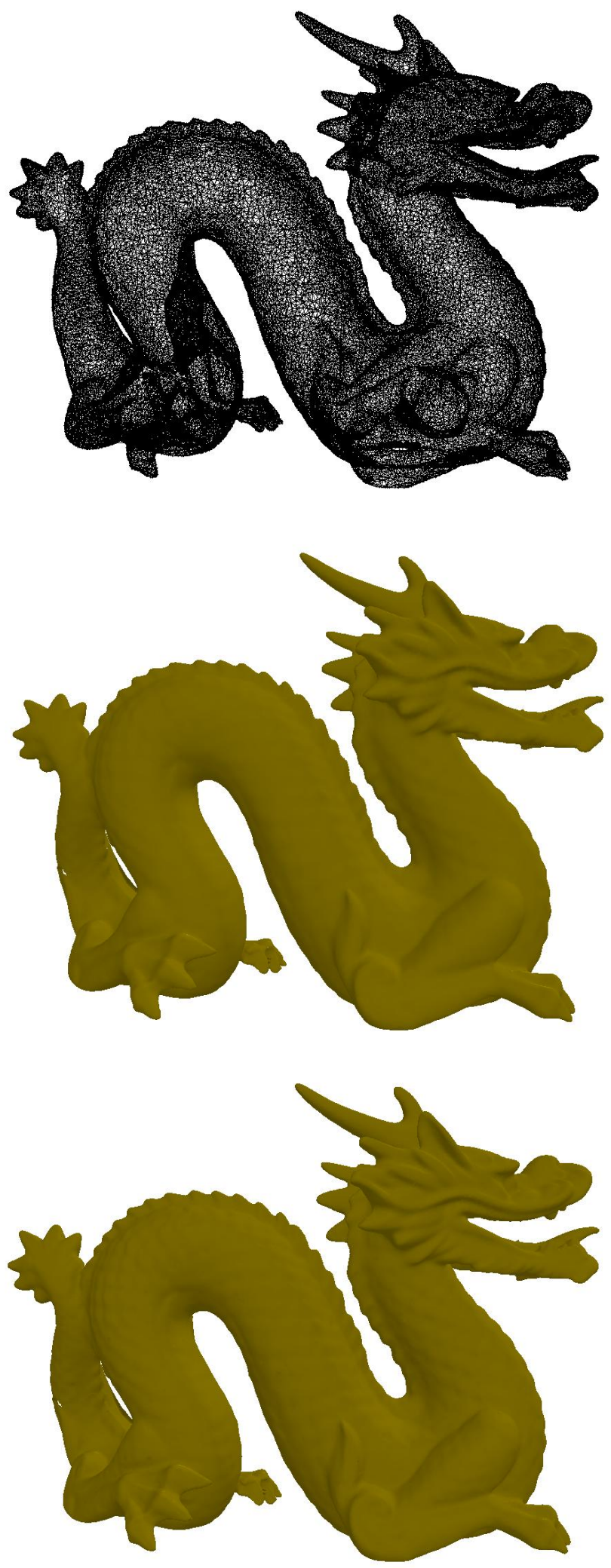

Figure 6: Dragon 

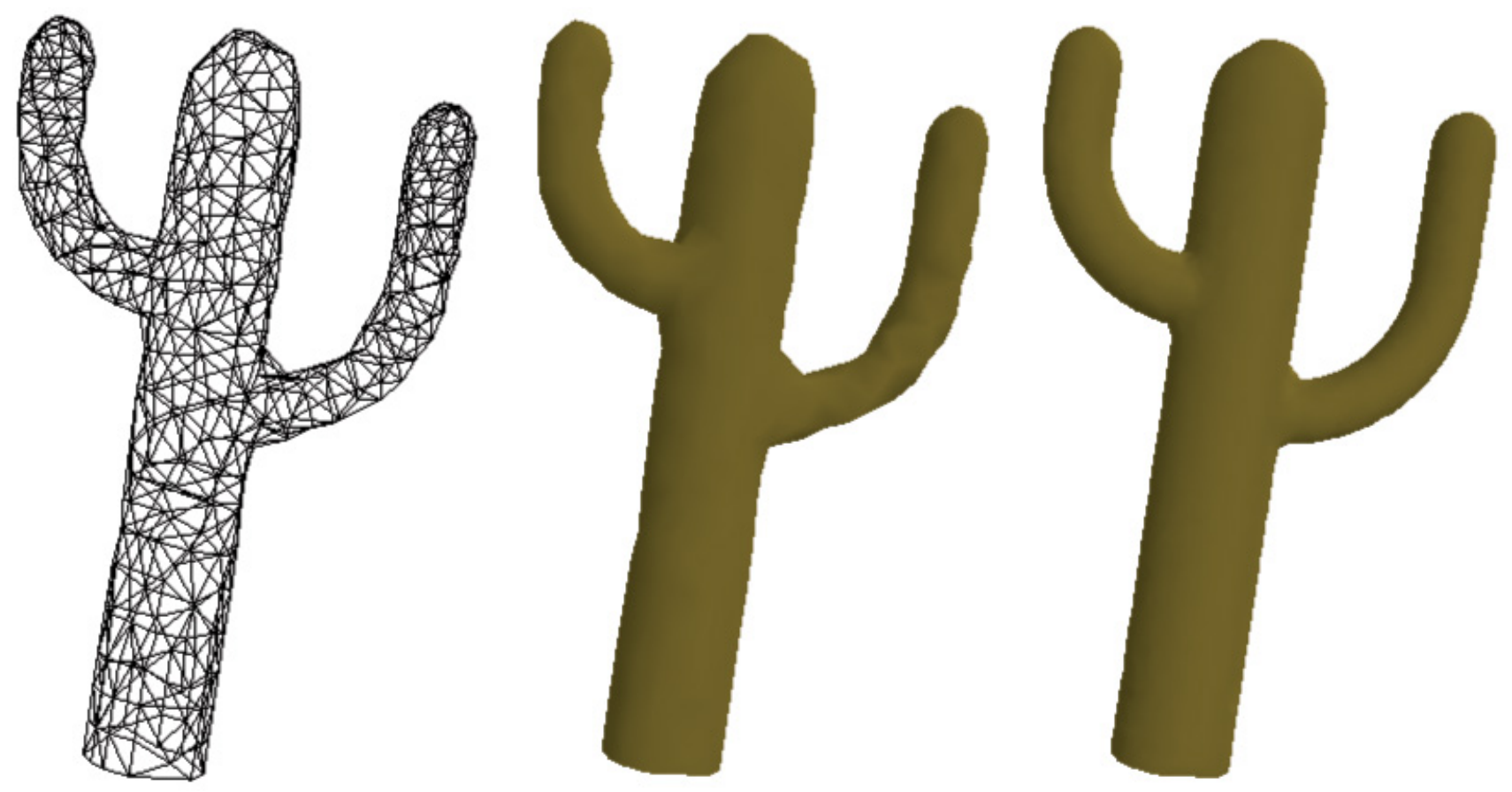

Figure 7: Cactus
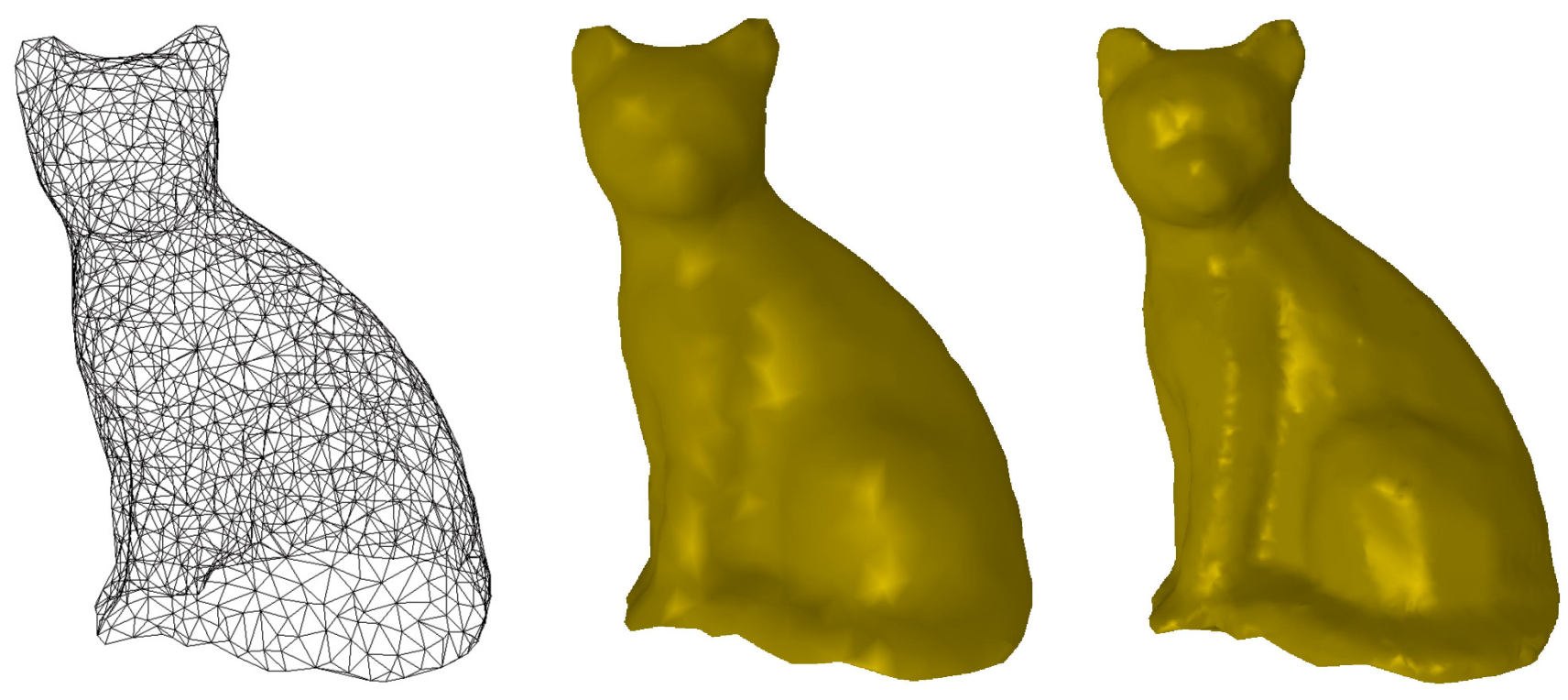

Figure 8: Cat 

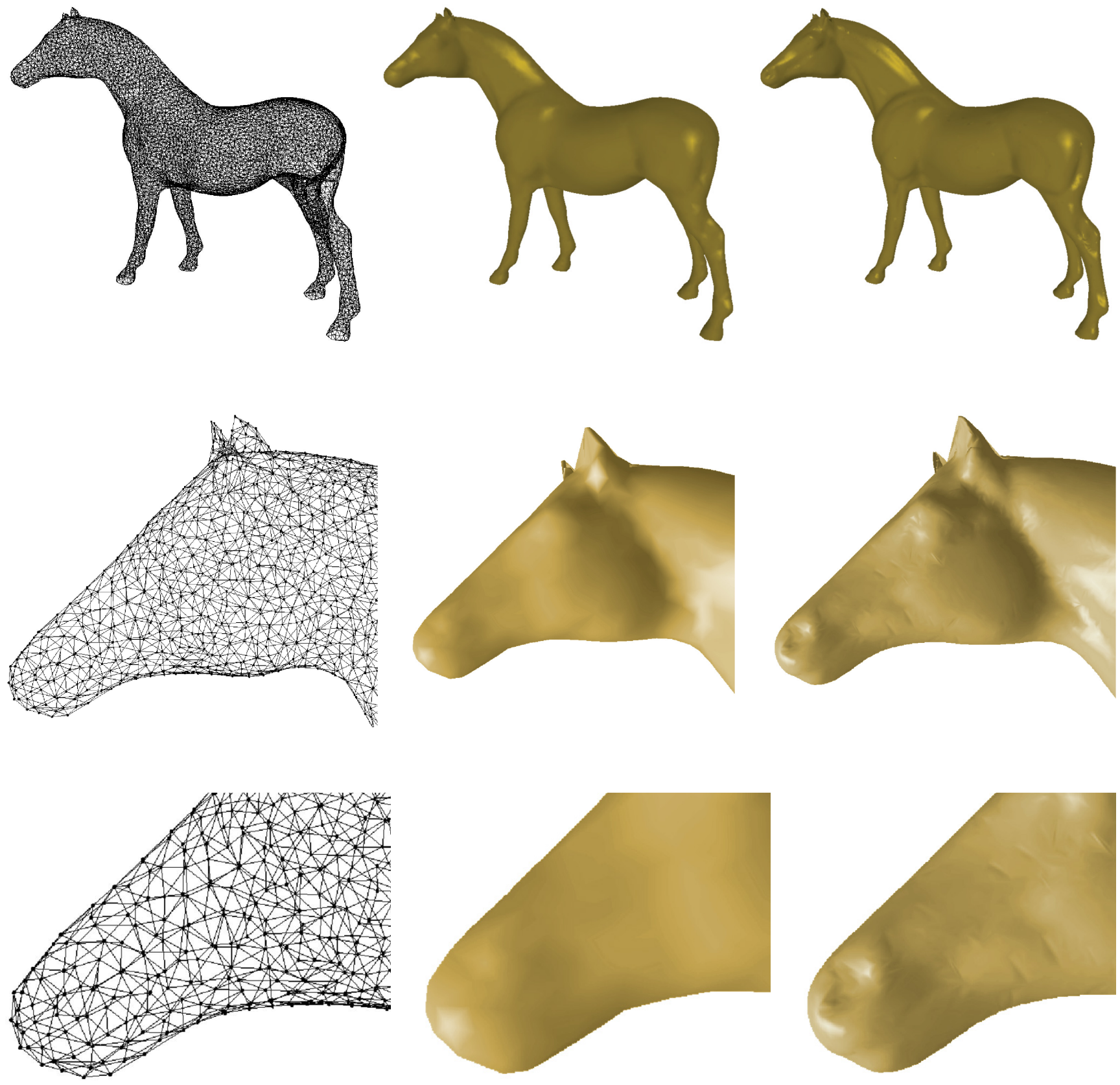

Figure 9: Horse 